PROCEEDINGS OF THE

AMERICAN MATHEMATICAL SOCIETY

Volume 132, Number 3, Pages 783-788

S 0002-9939(03)07102-8

Article electronically published on August 19, 2003

\title{
REAL RANK AND SQUARING MAPPINGS FOR UNITAL $C^{*}$-ALGEBRAS
}

\author{
A. CHIGOGIDZE, A. KARASEV, AND M. RØRDAM
}

(Communicated by David R. Larson)

\begin{abstract}
It is proved that if $X$ is a compact Hausdorff space of Lebesgue dimension $\operatorname{dim}(X)$, then the squaring mapping $\alpha_{m}:\left(C(X)_{\mathrm{sa}}\right)^{m} \rightarrow C(X)_{+}$, defined by $\alpha_{m}\left(f_{1}, \ldots, f_{m}\right)=\sum_{i=1}^{m} f_{i}^{2}$, is open if and only if $m-1 \geq \operatorname{dim}(X)$. Hence the Lebesgue dimension of $X$ can be detected from openness of the squaring maps $\alpha_{m}$. In the case $m=1$ it is proved that the map $x \mapsto x^{2}$, from the selfadjoint elements of a unital $C^{*}$-algebra $A$ into its positive elements, is open if and only if $A$ is isomorphic to $C(X)$ for some compact Hausdorff space $X$ with $\operatorname{dim}(X)=0$.
\end{abstract}

\section{INTRODUCTION}

A compact Hausdorff space $X$ is defined to have Lebesgue dimension $\leq m$ if for every closed subset $F$ of $X$, each continuous map $F \rightarrow S^{m}$ has a continuous extension $X \rightarrow S^{m}$.

Various types of ranks for (unital) $C^{*}$-algebras have been inspired by corresponding prototypes in the classical dimension theory of (compact) spaces, such as the one given above. While the Lebesgue dimension of a compact space has numerous equivalent formulations, the extensions of these equivalent formulations to noncommutative $C^{*}$-algebras most often differ. Examples of such ranks for $C^{*}$-algebras are the stable rank defined by Rieffel in [7, the real rank defined by Brown and Pedersen in [1, the analytic rank defined by Murphy in [4, the tracial rank defined by Lin in [3], the completely positive rank considered by Winter in [8], and the bounded rank defined in 2] (see also 6] for the definition of the exponential rank).

It was shown in 2 that a unital $C^{*}$-algebra $A$ has real rank at most $n$ if the squaring map $\left(x_{1}, \ldots, x_{n+1}\right) \mapsto \sum_{i=1}^{n+1} x_{i}^{2}$, from the set of $(n+1)$-tuples of selfadjoint elements to the set of positive elements in $A$, is open; and it was asked if the reverse also holds, in which case openness of the squaring maps would determine the real rank of the $C^{*}$-algebra.

In the present note we answer this question in the affirmative in the commutative case, and in the negative in the general (noncommutative) case. The latter, negative answer, follows from our result that the squaring map $x \mapsto x^{2}$ (from the set of

Received by the editors February 15, 2002 and, in revised form, October 28, 2002.

2000 Mathematics Subject Classification. Primary 46L05; Secondary 46L85, 54F45.

Key words and phrases. Real rank, bounded rank, Lebesgue dimension.

The first named author was partially supported by an NSERC research grant. 
selfadjoint elements to the set of positive elements) is open if and only if the $C^{*}$ algebra is commutative and of real rank zero. Hence the squaring map $x \mapsto x^{2}$ is not open for the $C^{*}$-algebra $M_{2}$ of 2 by 2 matrices, but this $C^{*}$-algebra has real rank zero.

\section{ACKNOWLEDGMENT}

The authors would like to thank A. N. Dranishnikov for useful conversations related to Proposition 2.2.

\section{RESUlTS}

For a $C^{*}$-algebra $A$ we use the standard notation $A_{\mathrm{sa}}$ and $A_{+}$to denote the set of all selfadjoint and the set of all positive elements of $A$, respectively. The real rank of a unital $C^{*}$-algebra $A$, denoted by $\mathrm{RR}(A)$, is in [1] defined as follows: For each nonnegative integer $n, \operatorname{RR}(A) \leq n$ if for every $(n+1)$-tuple $\left(x_{1}, \ldots, x_{n+1}\right)$ in $A_{\mathrm{sa}}$ and every $\varepsilon>0$, there exists an $(n+1)$-tuple $\left(y_{1}, \ldots, y_{n+1}\right)$ in $A_{\mathrm{sa}}$ such that $\sum_{k=1}^{n+1} y_{k}^{2}$ is invertible and $\sum_{k=1}^{n+1}\left\|x_{k}-y_{k}\right\|<\varepsilon$.

Let us say that a unital $C^{*}$-algebra $A$ has an open $m$-squaring map if the map $\alpha_{m}:\left(A_{s a}\right)^{m} \rightarrow A_{+}$, defined by $\alpha_{m}\left(x_{1}, \ldots, x_{m}\right)=\sum_{k=1}^{m} x_{k}^{2}$, is open. Observe that $\alpha_{m}$ is open at $\left(x_{1}, \ldots, x_{m}\right)$ if for every $\varepsilon>0$ there is $\delta>0$ such that for all $a \in A_{+}$ with $\left\|\sum_{k=1}^{m} x_{k}^{2}-a\right\|<\delta$ there is an $m$-tuple $\left(y_{1}, \ldots, y_{m}\right)$ in $A_{\text {sa }}$ with $\sum_{k=1}^{m} y_{k}^{2}=a$ and $\sum_{k=1}^{m}\left\|x_{k}-y_{k}\right\|<\varepsilon$.

For the reader's convenience we present a shorter proof of [2, Proposition 7.1].

Proposition 2.1. Let $A$ be a unital $C^{*}$-algebra. If the $(n+1)$-squaring map on $A$ is open, then $\operatorname{RR}(A) \leq n$.

Proof. Let $\left(x_{1}, \ldots, x_{n+1}\right)$ be an $(n+1)$-tuple of selfadjoint elements in $A$ and let $\varepsilon>0$. By openness of the $(n+1)$-squaring map there is $\delta>0$ and an $(n+1)$ tuple $\left(y_{1}, \ldots, y_{n+1}\right)$ of selfadjoint elements in $A$ such that $\sum_{k=1}^{n+1}\left\|x_{k}-y_{k}\right\|<\varepsilon$ and $\sum_{k=1}^{n+1} y_{k}^{2}=\sum_{k=1}^{n+1} x_{k}^{2}+\delta \cdot 1$, and the latter element is invertible (because each $x_{k}^{2}$ is positive).

Next, we prove the reverse of Proposition 2.1 in the commutative case.

Proposition 2.2. If $X$ is a compact space such that $\operatorname{dim} X \leq n$, then $C(X)$ has an open $(n+1)$-squaring map.

Proof. Let $f=f_{1}^{2}+\cdots+f_{n+1}^{2}$. Put $m_{i}=\sup f_{i}$ for $i=1, \ldots, n+1$ and let $m=\max \left\{m_{i}\right\}$. Fix $\varepsilon>0$ and let

$$
\delta=\min \left\{\frac{(\varepsilon / 3)^{4}}{m^{2}},\left(\frac{\varepsilon}{3}\right)^{2}\right\} \text { and } U=\left\{x \in X: f(x)>\left(\frac{\varepsilon}{3}\right)^{2}\right\} .
$$

Also, let $A=f^{-1}\left(\left[0,(\varepsilon / 3)^{2}\right]\right)$ and $S=f^{-1}\left((\varepsilon / 3)^{2}\right)$. Then $A$ and $S$ are closed subsets of $X$ such that $A=X \backslash U$ and $S \subseteq A$.

Now consider the diagonal product

$$
F(x)=\left(f_{1}(x), \ldots, f_{n+1}(x)\right): X \rightarrow \mathbb{R}^{n+1}
$$

and note that

$$
A=F^{-1}\left(B^{n+1}\right) \text { and } S=F^{-1}\left(S^{n}\right),
$$

where

$$
B^{n+1}=B^{n+1}(\mathbf{0}, \varepsilon / 3) \text { and } S^{n}=\partial B^{n+1}(\mathbf{0}, \varepsilon / 3) \text {. }
$$


Since $\operatorname{dim} A \leq \operatorname{dim} X \leq n$, the map $F \mid S: S \rightarrow S^{n}$ admits an extension $H: A \rightarrow$ $S^{n}$ (see, for instance, [5, Ch. 3, Theorem 2.2]). Let $h_{i}: A \rightarrow \mathbb{R}$ be the $i$-th component of the map $H$. Since $H(A) \subseteq S^{n}$, it follows that $h_{1}^{2}+\cdots+h_{n+1}^{2}=(\varepsilon / 3)^{2}$. Note also that since $H|S=F| S$ we have $h_{i}\left|S=f_{i}\right| S$ for each $i=1, \ldots, n+1$.

The last condition allows us to define for each $i=1, \ldots, n+1$ a continuous map $\widetilde{h_{i}}$ on $X$ by letting

$$
\widetilde{h_{i}}(x)= \begin{cases}f_{i}(x), & \text { if } x \in U, \\ h_{i}(x), & \text { if } x \in A .\end{cases}
$$

Observe that the function $\widetilde{h}=\widetilde{h}_{1}^{2}+\cdots+\widetilde{h}_{n+1}^{2}$ is strictly positive on $X$. Notice also that $\left.\widetilde{h}\right|_{U}=\left.f\right|_{U}$ and $\left.\widetilde{h}\right|_{A}=(\varepsilon / 3)^{2}$.

Next, take a positive function $g$ in $C(X)$ with $\|f-g\|_{\infty}<\delta$. Define a function $\lambda$ on $X$ by $\lambda(x)=(g(x) / \widetilde{h}(x))^{1 / 2}$. Note that $\lambda \geq 0$.

Now define $g_{i}$ for $i=1, \ldots, n+1$ on $X$ by the formula $g_{i}(x)=\widetilde{h}_{i}(x) \cdot \lambda(x)$. Clearly,

$$
\begin{aligned}
& g_{1}^{2}(x)+\cdots+g_{n+1}^{2}(x)=\left(\widetilde{h}_{1}(x) \cdot \lambda(x)\right)^{2}+\cdots+\left(\widetilde{h}_{n+1}(x) \cdot \lambda(x)\right)^{2} \\
& \quad=\lambda^{2}(x)\left(\widetilde{h}_{1}^{2}(x)+\cdots+\widetilde{h}_{n+1}^{2}(x)\right)=\frac{g(x)}{\widetilde{h}(x)} \cdot\left(\widetilde{h}_{1}^{2}(x)+\cdots+\widetilde{h}_{n+1}^{2}(x)\right)=g(x) .
\end{aligned}
$$

Next, let us show that $g_{i}$ is sufficiently close to $f_{i}$ for each $i=1, \ldots, n+1$. Indeed, since $\|f-g\|_{\infty}<\delta$ we conclude that for each $x \in A$ we have

$$
g(x)<f(x)+\delta<\left(\frac{\varepsilon}{3}\right)^{2}+\left(\frac{\varepsilon}{3}\right)^{2} .
$$

Since $g_{i}^{2} \leq g$ and $f_{i}^{2} \leq f$, the last inequality implies that

$$
\left|g_{i}(x)\right|<\sqrt{2\left(\frac{\varepsilon}{3}\right)^{2}}<2 \frac{\varepsilon}{3} \quad \text { and } \quad\left|f_{i}(x)\right| \leq \frac{\varepsilon}{3}
$$

for all $x \in A$. Hence

$$
\left|f_{i}(x)-g_{i}(x)\right| \leq\left|f_{i}(x)\right|+\left|g_{i}(x)\right|<\frac{\varepsilon}{3}+2 \frac{\varepsilon}{3}=\varepsilon
$$

as required.

Furthermore, if $x \in U$, then $\widetilde{h}(x)=f(x)$ and, consequently,

$$
\widetilde{h}(x)-\delta<g(x)<\widetilde{h}(x)+\delta .
$$

Hence

for $x \in U$. Since

$$
1-\frac{\delta}{\widetilde{h}(x)}<\frac{g(x)}{\widetilde{h}(x)}<1+\frac{\delta}{\widetilde{h}(x)}
$$

$$
\widetilde{h}(x)=f(x)>\left(\frac{\varepsilon}{3}\right)^{2} \text { for } x \in U \text { and } \delta \leq \frac{(\varepsilon / 3)^{4}}{m^{2}},
$$

we have (for $x \in U$ )

$$
1-\left(\frac{\varepsilon}{3 m}\right)^{2}=1-\frac{\frac{1}{m^{2}} \cdot\left(\frac{\varepsilon}{3}\right)^{4}}{\left(\frac{\varepsilon}{3}\right)^{2}}<\frac{g(x)}{\widetilde{h}(x)}<1+\frac{\frac{1}{m^{2}} \cdot\left(\frac{\varepsilon}{3}\right)^{4}}{\left(\frac{\varepsilon}{3}\right)^{2}}=1+\left(\frac{\varepsilon}{3 m}\right)^{2}
$$

and

$$
1-\left(\frac{\varepsilon}{3 m}\right)^{2}<\lambda^{2}(x)<1+\left(\frac{\varepsilon}{3 m}\right)^{2} .
$$


Consequently,

$$
\left|1-\lambda^{2}(x)\right|<\left(\frac{\varepsilon}{3 m}\right)^{2} .
$$

Since $\lambda(x) \geq 0$, this implies

$$
[1-\lambda(x)]^{2} \leq|1-\lambda(x)| \cdot|1+\lambda(x)|=\left|1-\lambda^{2}(x)\right|<\left(\frac{\varepsilon}{3 m}\right)^{2} .
$$

Therefore,

$$
|1-\lambda(x)| \leq \frac{\varepsilon}{3 m} \text { for any } x \in U
$$

Finally, we have

$$
\left|f_{i}(x)-g_{i}(x)\right|=|1-\lambda(x)| \cdot\left|f_{i}(x)\right|<\frac{\varepsilon}{3 m} \cdot m<\frac{\varepsilon}{3} \quad \text { for any } x \in U .
$$

This completes the verification of the fact that $\left|f_{i}(x)-g_{i}(x)\right|<\varepsilon$ for each $x \in X$ and any $i=1, \ldots, n+1$.

Corollary 2.3. Let $A$ be a unital $C^{*}$-algebra. Then the following conditions are equivalent:

(i) The squaring map $x \mapsto x^{2}$ from $A_{\mathrm{sa}}$ to $A_{+}$is open.

(ii) $A$ is commutative and $\operatorname{RR}(A)=0$.

(iii) $A$ is isomorphic to a $C^{*}$-algebra of the form $C(X)$ for a compact Hausdorff space $X$ with $\operatorname{dim} X=0$.

Proof. The equivalence of (ii) and (iii) follows from Gelfand's duality and 1, Proposition 1.1].

The implication (iii) $\Rightarrow$ (i) follows from Proposition 2.2

(i) $\Rightarrow$ (ii). Assume that (i) holds. Then $\operatorname{RR}(A)=0$ by Proposition 2.1. It remains to show that $A$ is commutative. Since $A$ is of real rank zero, it suffices to show that any two projections $p, q$ in $A$ commute.

Take the symmetry $s=p-(1-p)$. Then $s$ is selfadjoint and $s^{2}=1$. By openness of the squaring map there are selfadjoint elements $s_{n}$ in $A$ such that $\left\|s_{n}-s\right\| \rightarrow 0$ and $s_{n}^{2}=1+n^{-1} q$. Define $\varphi: \mathbb{R} \rightarrow \mathbb{R}$ by $\varphi(t)=\max \{0, t\}$. For each $n$, the element $\varphi\left(s_{n}\right)$ commutes with $s_{n}$, hence with $s_{n}^{2}$, and hence with $q$. Since $\varphi(s)=p$, we obtain

$$
p q-q p=\lim _{n \rightarrow \infty}\left(\varphi\left(s_{n}\right) q-q \varphi\left(s_{n}\right)\right)=0,
$$

as desired.

\section{RELATED COMMENTS AND OPEN PROBlems}

Existence of square roots. Suppose that $A$ is a unital $C^{*}$-algebra and that $x$ is a selfadjoint element in $A$. Does there exist a continuous square root $\rho_{x}=\rho: \Omega \rightarrow A_{\text {sa }}$ (i.e., $\rho(a)^{2}=a$ for all $a \in \Omega$ ) defined on an open neighborhood $\Omega \subseteq A_{+}$of $x^{2}$ such that $\rho\left(x^{2}\right)=x$ ? If this is true for all selfadjoint elements $a$ in $A$, then the equivalent conditions of Corollary 2.3 are satisfied.

Suppose that $A=C(X)$ for some 0-dimensional compact Hausdorff space $X$ (i.e., that the conditions of Corollary 2.3 are satisfied). Take a selfadjoint (i.e., realvalued) $f \in C(X)$, and suppose that there is a clopen set $U$ such that $f(x) \geq 0$ for 
all $x \in U$ and $f(x) \leq 0$ for all $x \in X \backslash U$. Then the function $\rho_{U}: C(X)_{+} \rightarrow C(X)_{\mathrm{sa}}$ defined by

$$
\rho_{U}(g)= \begin{cases}\sqrt{g(x)}, & x \in U, \\ -\sqrt{g(x)}, & x \in X \backslash U,\end{cases}
$$

is a continuous square root with $\rho_{U}\left(f^{2}\right)=f$. It is not clear to the authors if there are continuous square roots at arbitrary real-valued functions $f$ in $C(X)$.

In the case where $A=M_{n}$, the $C^{*}$-algebra of $n$ by $n$ matrices, if $x$ is a selfadjoint element and if $x^{2}$ has $n$ distinct eigenvalues, then there is a continuous square root $\rho$ with $\rho\left(x^{2}\right)=x$ defined on some neighborhood of $x^{2}$.

In the case where $A=M_{2}$, it follows from Corollary 2.3 (and its proof) that there is no continuous square root $\rho$ defined on a neighborhood of $I$ such that $\rho(I)=\operatorname{diag}(1,-1)$. It is easily checked explicitly that if $r$ is a (small) nonzero real number, then any square root of $\left(\begin{array}{ll}1 & r \\ r & 1\end{array}\right)$ is of the form $\left(\begin{array}{ll}a & s \\ s & a\end{array}\right)$, where $a$ and $s$ are real numbers satisfying $a^{2}+s^{2}=1$ and $2 a s=r$, and any such square root has distance at least 1 to $\operatorname{diag}(1,-1)$.

We end this note by listing some open problems related to openness of the squaring maps:

Question 1. Let $A$ be a unital $C^{*}$-algebra, let $m$ be a positive integer, and suppose that the squaring map $\alpha_{m}$ (defined above Proposition 2.1) is open. Does it follow that $\alpha_{n}$ is open for all $n \geq m$ ?

The answer to Question 1 is affirmative for commutative $C^{*}$-algebras by Propositions 2.1 and 2.2 The difficulty in this question lies in the fact that if $\Omega$ is an open subset of $A_{+}$and if $a \in A_{+}$, then $a+\Omega$ need not be open in $A_{+}$. (For instance, $1+A_{+}$is not open in $A_{+}$.)

Question 2. Are Propositions 2.1 and 2.2 valid also in the non-unital case? (For Proposition 2.2, this means that we will be talking about locally compact Hausdorff spaces rather than compact Hausdorff spaces.) What is the relationship between openness of $\alpha_{n}$ on a non-unital $C^{*}$-algebra $A$ and openness of $\alpha_{n}$ on its unitization?

Question 3. Are the squaring maps $\alpha_{m}$ open for all $m \geq 2$ when $A$ is a unital $C^{*}$-algebra of real rank zero?

Question 4. Does the class of $C^{*}$-algebras, for which the squaring map $\alpha_{2}$ is open, have any nice properties? More generally, are there any justifications for considering the rank of a $C^{*}$-algebra defined by openness of the squaring maps; and will this rank reflect any "dimension-like" properties of the $C^{*}$-algebra?

\section{REFERENCES}

[1] L. G. Brown, G. K. Pedersen, $C^{*}$-algebras of real rank zero, J. Functional Anal. 99 (1991), 131-149. MR 92m:46086

[2] A. Chigogidze, V. Valov, Bounded rank of $C^{*}$-algebras, Preprint math.OA/0109100 (2001).

[3] H. Lin, The tracial topological rank of $C^{*}$-algebras, Proc. London Math. Soc. 83 (2001), 199-234. MR 2002e: 46063

[4] G. J. Murphy, The analytic rank of a $C^{*}$-algebra, Proc. Amer. Math. Soc. 115 (1992), 741746. MR 92i: 46085

[5] A. R. Pears, Dimension Theory of General Spaces, Cambridge University Press, Cambridge, 1975. MR 52:15405

[6] N. C. Phillips, Simple $C^{*}$-algebras with the property weak (FU), Math. Scand. 69 (1991), 121-151. MR 93d:46121 
[7] M. A. Rieffel, Dimension and stable rank in the $K$-theory of $C^{*}$-algebras, Proc. London Math. Soc. 46 (1983), 301-333. MR 84g:46085

[8] W. Winter, Covering dimension for nuclear $C^{*}$-algebras, Schriftenreihe Math. Inst. Univ. Munster 3. Ser., 26, Heft 26, 95 pp., Univ. Munster, Munster, 2000. MR 2002c: 46134

Department of Mathematics and Statistics, University of Saskatchewan, McLean Hall, 106 Wiggins Road, Saskatoon, SK, S7N 5E6, Canada

E-mail address: chigogid@math.usask.ca

Department of Mathematics and Statistics, University of Saskatchewan, McLean Hall, 106 Wiggins Road, Saskatoon, SK, S7N 5E6, Canada

E-mail address: karasev@math.usask.ca

Department of Mathematics, University of Southern Denmark, Campusvej 55, 5230 Odense M, Denmark

E-mail address: mikael@imada.sdu.dk 